\title{
A Kirchhoff approximation-based numerical method to compute multiple acoustic scattering of a moving source
}

\author{
Nicolas J. Pignier ${ }^{1}$, Ciarán J. O’Reilly, Susann Boij \\ KTH Royal Institute of Technology, Department of Aeronautical and Vehicle Engineering, Teknikringen 8, 10044 Stockholm, Sweden \\ The Centre for ECO ${ }^{2}$ Vehicle Design at KTH, Teknikringen 8, 10044 Stockholm, Sweden
}

\begin{abstract}
Within the scope of a study of external noise propagation from moving ground vehicles, a numerical method is developed to compute the acoustic field emitted by a moving source in the presence of scattering objects such as roads, buildings or noise-shields. This method is developed with the purpose of being used in a vehicle design process and therefore it must have a low computational cost, which requires a certain number of approximations. The case of a fixed point source is studied first then the effect of a movement of the source is taken into account through the introduction of a retarded time. The acoustic source is assumed to be represented by one or many harmonic monopoles of possibly different frequency moving with a constant speed in a quiescent flow field. Scattering from nearby perfectly reflecting objects is computed through a Kirchhoff-Helmholtz integral equation applying the Kirchhoff approximation. A ray-surface intersection algorithm to compute shadow areas is proposed. The method is validated against analytical solutions and experimental results for a fixed source, and against a higher-order finite difference time-domain method for the multiple scattering of a moving source. Results are good and show that this method can potentially be used to predict urban noise.
\end{abstract}

Keywords: multiple scattering, moving source, Kirchhoff-Helmholtz integral, Kirchhoff approximation, urban noise

\section{Introduction}

Environmental noise is considered to be one of the main sources of local pollution. According to the European Commission, $30 \%$ of the European population may be exposed to annoyance and sleep disturbance related to environmental noise [1], causing a loss of at least 1.6 million Disability Adjusted Life Years every year. The external cost of noise in the European Union is estimated to reach $0.35 \%$ of the GDP. Environmental noise exposure is mainly related to traffic noise, as $24.3 \%$ of the European population - EU25 Member States, Candidate Countries and countries of the European Economic Area (EEA)-lives within $500 \mathrm{~m}$ of a road with a traffic of more than 3 million vehicles per year and $2.6 \%$ within $500 \mathrm{~m}$ of a railway with a traffic of more than 60000 trains per year [2]. People are mostly exposed to traffic noise in urban area, where the sound field generated by passing vehicles is strongly affected by the surrounding buildings. Therefore there is a need for numerical models to compute the sound field in such conditions. This paper presents a numerical method to predict the sound field generated by a moving source in presence of large reflecting objects, with a focus on the sound in the area surrounding the source itself. There exist several empirical or semi-empirical models to predict road and railway traffic noise [3, 4, 5, 6, 7, 8]

\footnotetext{
http://dx.doi.org/10.1016/j.apacoust.2015.03.016

Email address: pignier@kth. se (Nicolas J. Pignier)

${ }^{1}$ Corresponding author
}

for which Garg and Maji published a comparative review [9]. These methods are mainly used by consultants, land planners and highway engineers and focus on community noise [10]. They include both source and propagation models, which are shifting from empirical models to more physical models as the computational resources increase [9]. Together with the development of these methods, propagation models for the wave emitted by a point source in an urban environment have been studied, using methods such as the boundary element method (BEM), ray-tracing algorithms or geometrical acoustic methods combined with diffraction models [11]. Another method worth mentioning is the extended Fourier pseudospectral time-domain method based on the linearized Euler equations developed by Hornikx [12]. These methods are solving different equations and have varying degrees of accuracy. The BEM is expected to give accurate results, but it can become very computationally demanding for high frequencies [13]. Heutschi presented in 2009 a method to compute multiple coherent reflections in urban environment based on the Kirchhoff approximation, which is part of the Swiss railway noise calculation sonRAIL [14]. The work presented here develops a similar idea with a different approach, extended to the case of a moving source. It can be seen as a complementary approach to the methods cited above, for which the extension to a movement of the source in a non quasi-static fashion is not obvious. Methods to compute propagation from moving sources and scattering by rigid surfaces can also be found in the field of aeroacoustics, even though they have been developed to solve a different problem. One example 
is the Fast Scattering Code developed by Tinetti [15] that could potentially be applicable to our problem.

This work is presented in three sections. In the first section we introduce the Kirchhoff-Helmholtz equation, the Kirchhoff approximation and its application to acoustic scattering. In the next section we explain how this equation can be extended to the case of multiple scattering and to the case of a moving source. We propose a method to compute the so-called shadow areas. In the last section we present three cases of validation: the scattering of a plane wave by a rigid sphere, the scattering of a spherical wave by a rigid thin panel and the scattering of a moving source by two tall buildings.

\section{The Kirchhoff-Helmholtz equation, The Kirchhoff ap- proximation, and its application to acoustic scattering}

This section introduces the Kirchhoff-Helmholtz equation and the Kirchhoff approximation applied to the computation of the scattering of a spherical sound wave by a rigid object. The equations in this section are well-known but are presented here again for the convenience of the reader. In what follows sound propagation is considered in a homogeneous, isotropic, linear, three dimensional quiescent fluid. It is assumed that the acoustic pressure is given by the solution of the wave equation everywhere within the boundaries of the domain. The equations being linear, this study can be limited to the case of a single point source, the case of multiple point sources can then be obtained by summation. The variable $p$ refers to the time-domain acoustic pressure and $\hat{p}$ to the frequency-domain acoustic pressure. The acoustic pressures $p$ and $\hat{p}$ are linked by the relation $p(\mathbf{x}, t)=\hat{p}(\mathbf{x}) \exp (i \omega t)$ where $\omega$ is the angular frequency of the source.

\subsection{Kirchhoff-Helmholtz equation}

Let us consider a fixed object of arbitrary shape enclosed by a surface $\mathcal{S}$ in a quiescent flow field and a fixed harmonic monopole point source located at $\mathbf{y}$ outside $\mathcal{S}$. The Sommerfeld radiation condition is supposed to be fulfilled at infinity. The acoustic pressure $\hat{p}$ at an arbitrary point $\mathbf{x}$ located outside $\mathcal{S}$ and different from $\mathbf{y}$ can be expressed as

$$
\hat{p}(\mathbf{x})=\hat{p}_{\text {inc }}(\mathbf{x})+\hat{p}_{s c}(\mathbf{x})
$$

where $\hat{p}_{\text {inc }}$ is given by

$$
\hat{p}_{\text {inc }}(\mathbf{x})=\hat{S}_{0} \hat{G}(\mathbf{x}, \mathbf{y})
$$

and where $\hat{p}_{s c}$ is expressed through the Kirchhoff-Helmholtz equation as

$$
\hat{p}_{s c}(\mathbf{x})=\oiint_{\mathcal{S}}(\hat{p}(\mathbf{z}) \nabla \hat{G}(\mathbf{z}, \mathbf{x})-\hat{G}(\mathbf{z}, \mathbf{x}) \nabla \hat{p}(\mathbf{z})) \cdot \mathbf{n}_{\text {out }} d S_{z} .
$$

In Eq. (3), $\mathbf{z}$ is the variable on which the integral is performed, $d S_{z}$ is the elementary volume around $\mathbf{z}, \hat{S}_{0}$ is the amplitude of the source, $\mathbf{n}_{\text {out }}$ is the outward normal to the surface $\mathcal{S}$ and $\hat{G}$ is the free field Green function given by

$$
\hat{G}(\mathbf{x}, \mathbf{y})=\hat{G}(\mathbf{r})=\frac{1}{4 \pi r} e^{-i \mathbf{k r}} \text { with } \mathbf{r}=\mathbf{x}-\mathbf{y}
$$

where $r=|\mathbf{r}| . \hat{G}(\mathbf{x}, \mathbf{y})$ is the solution of the inhomogeneous Helmholtz equation $\left(\nabla^{2}+k^{2}\right) \hat{G}(\mathbf{x}, \mathbf{y})=-\delta(\mathbf{x}-\mathbf{y})$. Making the assumption of rigid objects, the second term in the integral in equation (3) reduces to zero since the acoustic pressure on its surface $\mathcal{S}$ satisfies the hard wall boundary condition $\nabla \hat{p} \cdot \mathbf{n}_{\text {out }}=$ 0 .

\subsection{The Kirchhoff approximation}

The Kirchhoff-Helmholtz integral equation presented in Eq. (3) is not in an explicit form. Indeed, the values of acoustic pressure on the surface of the object are affected by the scattered field itself. This equation can be used as such in a BEM, but it can have a very high computational cost for high frequencies. A way to lower the cost of the computation is to approximate the values of the surface pressure by using the Kirchhoff approximation. This assumption was originally derived to compute diffraction of a plane wave propagating through an aperture, and the formulation was the following [16]: on the integrated surface on the transmission side, the pressure field is assumed to be equal to the incident pressure field in the aperture and zero outside. This assumption can be extended to be used for the study of scattering by rigid objects, in which case the pressure value inside the Kirchhoff-Helmholtz integral is assumed to be equal to two times the incident pressure value on the surface for the insonified parts and zero in the shadowed parts. In other words, diffraction around the object is neglected in the process of surface pressure evaluation as well as reflection from one part of the object to another. This approximation is therefore a priori valid when these effects are negligible. This is more likely to be true for objects of convex shape at high frequency. A consequence of this approximation is that the shape of the object in the shadow area has no influence on the sound field. Using the Kirchhoff approximation on the expression for the scattered pressure yields

$$
\hat{p}_{s c}(\mathbf{x})=\oiint_{\mathcal{S}} \hat{p}_{\text {surf }}(\mathbf{z}) \nabla \hat{G}(\mathbf{z}, \mathbf{x}) \cdot \mathbf{n}_{\mathbf{o u t}} d S_{z}
$$

where

$$
\hat{p}_{\text {surf }}(\mathbf{z})=2 \hat{p}_{\text {inc }}(\mathbf{z}) \mathcal{D}(\mathbf{z}, \mathbf{y})
$$

where $\mathbf{y}$ is the position of the source. In equation (6) we have introduced a function $\mathcal{D}$ which accounts for shadow domains. $\mathcal{D}$ is defined as follows

$$
\mathcal{D}(\mathbf{x}, \mathbf{y})=\left\{\begin{array}{l}
0 \text { if the line segment }] \mathbf{x y}[\text { has an intersection } \\
\text { with at least one of the surface elements } \\
\text { constituting the object, } \\
1 \text { otherwise. }
\end{array}\right.
$$

and is evaluated with the method explained in section 3.1, assuming that the surface is meshed with triangular elements.

\subsection{Validity of the Kirchhoff approximation}

The validity of the Kirchhoff approximation has drawn a lot of attention in the past due to its lack of rigorous mathematical proof. Cox and Lam [17] compared different methods to evaluate the scattering from simple rigid plane and curved panels and 
compared them with measurements. The Kirchhoff-Helmholtz equation simplified with the Kirchhoff approximation had similar level of accuracy as a boundary element method for small scattering angles with a much faster computation time as the integral is computed directly whereas the BEM necessitates a matrix inversion. It was observed that the accuracy gets better with smaller angles of scattering and higher frequencies. A good accuracy of the approximation has also been observed by Foote and Francis [18] for high frequencies in far-field backscattering of sound by fishes. They noticed that the BEM and the Kirchhoff approximation gave similar results for Helmholtz numbers $k a>4$, where $a$ is the characteristic size of the scattering object. Eaton [19] also noted that results were good for $k a>5$ for the computation of backscattering from curved surface.

Limitations to the Kirchhoff approximation are found in low frequency scattering, high angles and for non convex scattering objects. Jebsen and Medwin [20] showed that the Kirchhoff approximation led to mistakes at grazing angles in the computation of the backscattering from a semi-infinite plate and from a rigid plane wedge. Wirgin [21] observed that the accuracy of the Kirchhoff approximation diminishes with increasing angle of incidence on a sinusoidal grating and that it led to erroneous results when multiple scattering effects on the surface of the object are not negligible.

To sum up, the Kirchhoff approximation can lead to accurate results with a moderate computation time, limitations being that the frequency has to be sufficiently high and the angle of scattering sufficiently low. The problem presented here is addressing mostly high frequencies. Indeed, the hearing range extends from about $20 \mathrm{~Hz}$ to $20 \mathrm{kHz}$ and for a sound wave of frequency higher than $20 \mathrm{~Hz}$ scattered by a building of characteristic size $10 \mathrm{~m}, \mathrm{ka}$ is greater than 4 . Moreover, this study focuses on the sound field on the side of the source so the propagation phenomena behind the buildings are not the main interest. Within this scope the Kirchhoff approximation seems applicable to our purpose.

\section{Implementation for multiple scattering and a moving source}

In this section, we explain how the Kirchhoff-Helmholtz integral equation is implemented to compute reflections from multiple objects, first in the case of a fixed source then for a moving source. A method to compute shadow domains is also presented in an introductory part, as a necessary tool to be used in the method.

\subsection{Computation of shadow domains}

In order to evaluate numerically the acoustic pressure on the surfaces of the scattering objects with the Kirchhoff approximation, it is necessary to know which parts of these objects belong to the shadow domain, defined here in terms of geometrical acoustics as the domain not directly reached by rays of sound from the source or from other objects. An algorithm is proposed to compute these shadow domains, but other methods could also be used.
The position of the source emitting the wave reaching a point $\mathbf{x}$ at $t$ is given by $\mathbf{y}\left(t_{e}\right)$, where $t_{e}$ is the retarded time. $\mathbf{x}$ is in the shadow domain at $t$ if the ray between $\mathbf{y}\left(t_{e}\right)$ and $\mathbf{x}$ is intercepted by a boundary. Assuming that the object surfaces are discretized into triangles for the numerical evaluation of the integrals, this problem comes down to the problem of knowing whether a line segment ] $\mathbf{x y}$ [ between two points in a 3D space has an intersection with a triangle $\mathcal{T}$ or not. In order to do that,

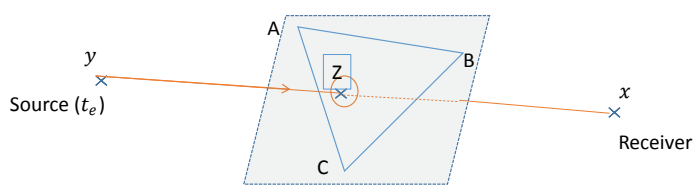

Figure 1: Intersection of a ray with a triangle in 3D space

we define the projection $\mathbf{Z}$ of the point $\mathbf{y}$ on the plane containing the triangle as explained in Figure 1 . If $\mathbf{Z}$ belongs to $\mathcal{T}$ then there is intersection and $\mathbf{x}$ belongs to the shadow domain. $\mathbf{Z}$ belongs to $\mathcal{T}$ if and only if $\mathbf{Z}$ can be expressed as a weighted sum of the vertices $\mathbf{A}, \mathbf{B}$ and $\mathbf{C}$, all weights being positive.

$$
\mathbf{Z} \in \mathcal{T} \Leftrightarrow \exists\{\alpha, \beta, \gamma\} \in \mathfrak{R}_{+}^{3} \backslash \mathbf{Z}=\alpha \mathbf{A}+\beta \mathbf{B}+\gamma \mathbf{C}
$$

In practice, we define $\mathbf{Z}$ then we calculate the weights $\alpha, \beta$ and $\gamma$ such as $\mathbf{Z}=\alpha \mathbf{A}+\beta \mathbf{B}+\gamma \mathbf{C}$ and we check if these weights are all positive.

This ray-intersection computation needs to be done a repeated number of times during the computation process. The higher the frequency of the source, the finer the mesh on the buildings and the higher the computational cost. The latter also increases with the complexity of the source - assuming the source can be modelled by a certain number of point sources of single frequency. Indeed, parts of the process have to be repeated for each point source. Increasing the number of objects in the field leads to an increase of the computational cost too. The computation of the shadow domains then becomes the limiting step, and a considerable gain in speed can be achieved by implementing an efficient method. In this study, to limit the number of calculations to perform, a filter is implemented to remove all obviously non-intersecting elements from the loop. The filter acts in the following way. Two planes are defined perpendicular to $[\mathbf{x y}]$ and passing by $\mathbf{x}$ and $\mathbf{y}$. The region between these two planes contains all potentially intersecting elements, therefore the elements located outside this region can be removed from the list of possible candidates for intersection. Then all projection points $Z$ are defined and the elements are ranked by increasing distance from their center node to the corresponding projected point $Z$, following the idea that for an element intersecting the ray, the projected point $Z$ will be close to its center node. Then the algorithm of intersection computation starts from the element for which the center node is closest to its projection point, and it stops as soon as an intersecting element is found, thus avoiding to perform unnecessary computations. To express the shadow domains mathematically in the follow- 
ing sections, a function $\mathcal{D}^{I}$ is introduced as

$$
\mathcal{D}^{I}(\mathbf{x}, \mathbf{y})=\left\{\begin{array}{l}
0 \text { if the line segment }[\mathbf{x y}] \text { has an } \\
\text { intersection with at least one of } \\
\text { the objects belonging to ensemble I, } \\
1 \text { otherwise. }
\end{array}\right.
$$

and is computed using the method described in this section. This function is an extension of the function $D$ defined in Eq. (7) to the case of multiple objects and multiple scattering. The brackets are now closed to indicate that the points $\mathbf{x}$ and $\mathbf{y}$ themselves have to be included in the computation of intersections.

\subsection{Formulation in case of multiple objects}

When there are more than one object in the field, the expression for the scattered pressure given by Eq. (5) has to be adapted. Under the same assumptions as in Section 2.1. The pressure at the receiver can be expressed as

$$
p(\mathbf{x}, t)=p_{\text {inc }}(\mathbf{x}, t)+\sum_{i=1}^{N} p_{s c, r}^{(i)}(\mathbf{x}, t)
$$

where $N$ is the number of objects and $p_{s c, r}^{(i)}$ is the pressure field scattered by the object $i$, given by

$$
p_{s c, r}^{(i)}(\mathbf{x}, t)=\oiint_{\mathcal{S}_{i}} p_{s u r f}^{(i)}(\mathbf{z}, t) \nabla \hat{G}(\mathbf{z}, \mathbf{x}) \cdot \mathbf{n}_{\text {out }} d S_{z} .
$$

In this expression surface pressures $p_{\text {surf }}^{(i)}$ of all objects are coupled and the exact solution to this equation implies an infinite number of reflections between the objects. Each object sees the other objects as sources that will contribute to the evaluation of the pressure values on its surface, and an infinite number of successive updates of the surface pressures is needed to lead to the solution. The infinite series arising are too expensive to compute without truncation, especially for high frequencies. In this paper only a finite number of reflections are considered, implying that multiple scattering effects over a certain level are negligible. The steps and assumptions needed to evaluate the surface pressures $p_{\text {surf }}^{(i)}$ for increasing levels of scattering are described in the following paragraphs.

A single level of scattering. If a single level of scattering is of interest, surface pressures are only evaluated once and only the wave coming directly from the source contributes to their value. Masking effects of the other objects on the surface of a given object are accounted for under the hypothesis of geometrical acoustics. In other words, surface pressure values do not account for scattering by other objects but only for geometrical masking by these objects. Only the insonified parts of the surfaces of all objects have a non-zero pressure value, all surfaces in the shadow areas have zero pressure value. $p_{\text {surf }}^{(i)}$ is evaluated through

$$
p_{\text {surf }}^{(i)}(\mathbf{z}, t)=2 p_{\text {inc }}^{(i)}(\mathbf{z}, t) \mathcal{D}^{\{1 . . N\}}(\mathbf{z}, \mathbf{y})
$$

where $\mathbf{y}$ is the position of the source and where $\mathcal{D}^{I}$ is defined by Eq. 93. In $\mathcal{D}^{\{1 \ldots N\}}$ all the objects are taken into account in the calculation of ray-surface intersection, including the object on which the surface pressure is evaluated.

The acoustic pressure at the listener is then computed using Eq. 10 where $p_{s c, r}^{(i)}$ is given by

$$
p_{s c, r}^{(i)}(\mathbf{x}, t)=\oiint_{\mathcal{S}_{i}} p_{s u r f}^{(i)}(\mathbf{z}, t) \nabla \hat{G}(\mathbf{z}, \mathbf{x}) \cdot \mathbf{n}_{\text {out }} \mathcal{D}^{\{1 \ldots N\} \backslash i}(\mathbf{x}, \mathbf{z}) d S_{z}
$$

$\mathcal{D}^{\{1 \ldots N\} \backslash i}$ expresses the hypothesis that only the equivalent sources for which a ray of sound reaches the listener contribute to the acoustic pressure at the listener. The interpretation of the Kirchhoff approximation in terms of equivalent Huygens' sources is described in Section 3.3 .

Two levels of scattering. If two or more levels of scattering are needed, then surface pressures have to account for the pressure from the source and the pressure scattered by the other objects. The surface pressures $p_{\text {surf }}^{(i, 1)}$ and $p_{\text {surf }}^{(i, 2)}$ on the object $i$ contributing to the 1 st and 2 nd levels of scattering are evaluated through

$$
\left[\begin{array}{rl}
p_{\text {surf }}^{(i, 1)}(\mathbf{z}, t) & =2 p_{\text {inc }}^{(i)}(\mathbf{z}, t) \mathcal{D}^{i}(\mathbf{z}, \mathbf{y}) \\
p_{\text {surf }}^{(i, 2)}(\mathbf{z}, t) & =\sum_{j=1, j \neq i}^{N} \oiint_{\mathcal{S}_{j}} p_{\text {surf }}^{(j, 1)}(\mathbf{z}, t) \nabla \hat{G}(\mathbf{z}, \mathbf{x}) . \mathbf{n}_{\text {out }} \\
\mathcal{D}^{\{1 \ldots N\} \backslash j}(\mathbf{x}, \mathbf{z}) d S_{z}
\end{array}\right.
$$

In $\mathcal{D}^{\{1 \ldots N\} \backslash j}$ all objects are taken into account in the calculation of ray-surface intersection, except the object from which the scattered wave comes. In $\mathcal{D}^{i}$ only the object on which the surface pressure is evaluated is included in the ray-surface intersection calculation, given that the masking effect of one object on another is accounted for during the update of the surface pressure values by the scattered field from this object.

The acoustic pressure at the listener is then computed using Eq. 10. where $p_{s c, r}^{(i)}=p_{s c, r}^{(i, 1)}+p_{s c, r}^{(i, 2)}$ with

$$
\left[\begin{array}{rl}
p_{s c, r}^{(i, 1)}(\mathbf{x}, t) & =\oiint_{\mathcal{S}_{i}} p_{\text {surf }}^{(i, 1)}(\mathbf{z}, t) \nabla \hat{G}(\mathbf{z}, \mathbf{x}) . \mathbf{n}_{\mathbf{o u t}} \\
p_{s c, r}^{(i, 2)}(\mathbf{x}, t) & =p_{s c, r}^{(i, 1)}(\mathbf{x}, t)+\oiint_{\mathcal{S}_{i}} p_{\text {surf }}^{(i, 2)}(\mathbf{z}, t) \nabla \hat{G}(\mathbf{z}, \mathbf{x}) \cdot \mathbf{n}_{\text {out }} \\
\mathcal{D}^{\{1 \ldots N\} \backslash i}(\mathbf{x}, \mathbf{z}) d S_{z}
\end{array}\right.
$$

Three or more levels of scattering. If $Q \geq 3$ levels of scattering are to be computed, then surface pressures corresponding to each level of scattering have to be calculated as detailed in Eq. 16.

$$
\left[\begin{array}{rl}
p_{\text {surf }}^{(i, 1)}(\mathbf{z}, t) & =2 p_{\text {inc }}^{(i)}(\mathbf{z}, t) \mathcal{D}^{i}(\mathbf{z}, \mathbf{y}) \\
p_{\text {surf }}^{(i, q)}(\mathbf{z}, t) & =\sum_{j=1, j \neq i}^{N} \oiint_{\mathcal{S}_{j}} p_{\text {surf }}^{(j, q-1)}(\mathbf{z}, t) \nabla \hat{G}(\mathbf{z}, \mathbf{x}) \cdot \mathbf{n}_{\text {out }} \\
p_{\text {surf }}^{(i, Q)}(\mathbf{z}, t) & =\sum_{j=1, j \neq i}^{N} \oiint_{\mathcal{S}_{j}} p_{\text {surf }}^{(j, Q-1)}(\mathbf{z}, t) \nabla \hat{G}(\mathbf{z}, \mathbf{x}) \cdot \mathbf{n}_{\text {out }} \\
\mathcal{D}^{\{1 \ldots N\} \backslash j}(\mathbf{x}, \mathbf{z}) d S_{z}
\end{array}\right.
$$

where $p_{\text {surf }}^{(i, q)}$ designates the value of the surface pressure on object $i$ contributing to the $\mathrm{q}^{\text {th }}$ level of scattering. The number of levels $Q$ is chosen depending on the number of scattering objects, on their configuration and on the desired order of accuracy.

The acoustic pressure at the listener is then computed using Eq. 
10 where $p_{s c, r}^{(i)}=\sum_{i=1}^{Q} p_{s c, r}^{(i, q)}$ with

$$
\begin{gathered}
q=1, \quad p_{s c, r}^{(i, 1)}(\mathbf{x}, t)=\oiint_{\mathcal{S}_{i}} p_{s u r f}^{(i, 1)}(\mathbf{z}, t) \nabla \hat{G}(\mathbf{z}, \mathbf{x}) \cdot \mathbf{n}_{\text {out }} \\
q \geq 2, \quad p_{s c, r}^{(i, q)}(\mathbf{x}, t)=p_{s c, r}^{(i, q-1)}(\mathbf{x}, t)+\oiint_{\mathcal{S}_{i}} p_{s u r f}^{(i, q)}(\mathbf{z}, t) \nabla \hat{G}(\mathbf{z}, \mathbf{x}) \\
p_{s c, r}^{(i, Q)}(\mathbf{x}, t)=p_{s c, r}^{(i, Q-1)}(\mathbf{x}, t)+\oiint_{\mathcal{S}_{i}} p_{\text {surf }}^{(i, Q)}(\mathbf{z}, t) \nabla \hat{G}(\mathbf{z}, \mathbf{x}) \cdot \mathbf{n}_{\text {out }} \\
\mathcal{D}^{\{1 \ldots N\} \backslash i}(\mathbf{x}, \mathbf{z}) d S_{z}
\end{gathered}
$$

The whole process is described in Figure (2) in the case of 2 objects and two levels of scattering.

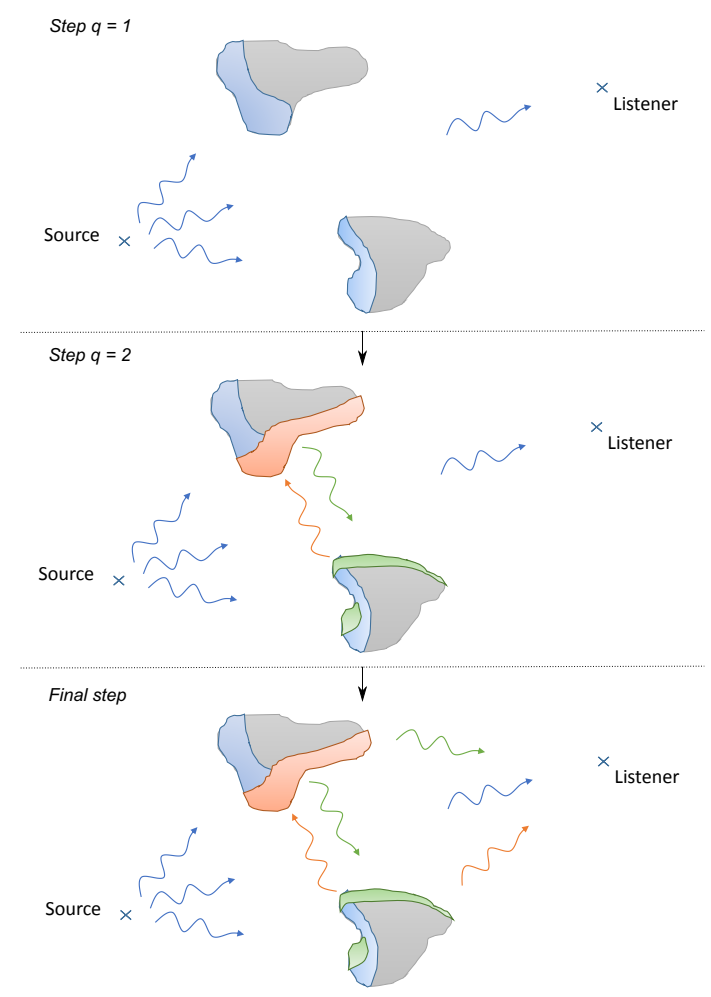

Figure 2: Computation process for 2 objects and 2 levels of scattering. Step $q=1$ : Incident pressure from the source is computed on the objects and on the observer. Illuminated areas where the surface pressure values are updated are represented in color.

Step $q=2$ : Surface pressure values on each object for the $2^{\text {nd }}$ level of scattering are computed from the scattered pressure from the other object using the suface pressure values of step 1.

Final step: Eventually acoustic pressure at the observer is computed by addition of the incident pressure wave and the scattered pressure waves from the two objects, for the two levels of scattering.

\subsection{Adaptation of the Kirchhoff-Helmholtz equation to a mov- ing point source}

If the source is moving with a constant subsonic speed $v_{0}$, as considered in this paper, then the movement of the source during the propagation from the source to the listener has to be accounted for. The acoustic incident pressure from the moving source is expressed as

$$
p(\mathbf{x}, t)=\frac{\hat{S}_{0}}{4 \pi r\left(t_{e}\right)\left|1-M_{r}\left(t_{e}\right)\right|} e^{i \omega_{0} t_{e}}
$$

where $t_{e}$ is the so-called retarded time solution of $c_{0}\left(t-t_{e}\right)=$ $r\left(t_{e}\right)$ and $\mathbf{r}\left(t_{e}\right)=\mathbf{x}-\mathbf{y}\left(t_{e}\right)$ [22]. $t_{e}$ corresponds to the time at which the pressure wave reaching the receiver at $t$ is emitted by the source. $M_{r}=\mathbf{M} \cdot \mathbf{e}_{\mathbf{r}}=M \cos \theta$ is the projection of the source Mach number $\left(\mathbf{M}=\mathbf{V} / c_{0}\right)$ in the direction towards the observer, and $\mathbf{e}_{\mathbf{r}}=\mathbf{r} / r$.

To express the scattered pressure through a Kirchhoff integral, we need to modify Eq. (5). Indeed the Kirchhoff-Helmholtz equation leading to Eq. (5) has been derived in the frequency domain and is therefore not applicable as it is in the case of a moving source. An intuitive approach to modify this equation is presented here.

Eq. (5) can be interpreted in terms of Huygens' sources: the presence of the object is replaced by a certain number of equivalent sources on its surface as explained in Figure 3. Due to the Doppler effect, each of these point sources receives and emits a wave of frequency $\omega_{e}$, where $\omega_{e}$ is a function of the position and time.
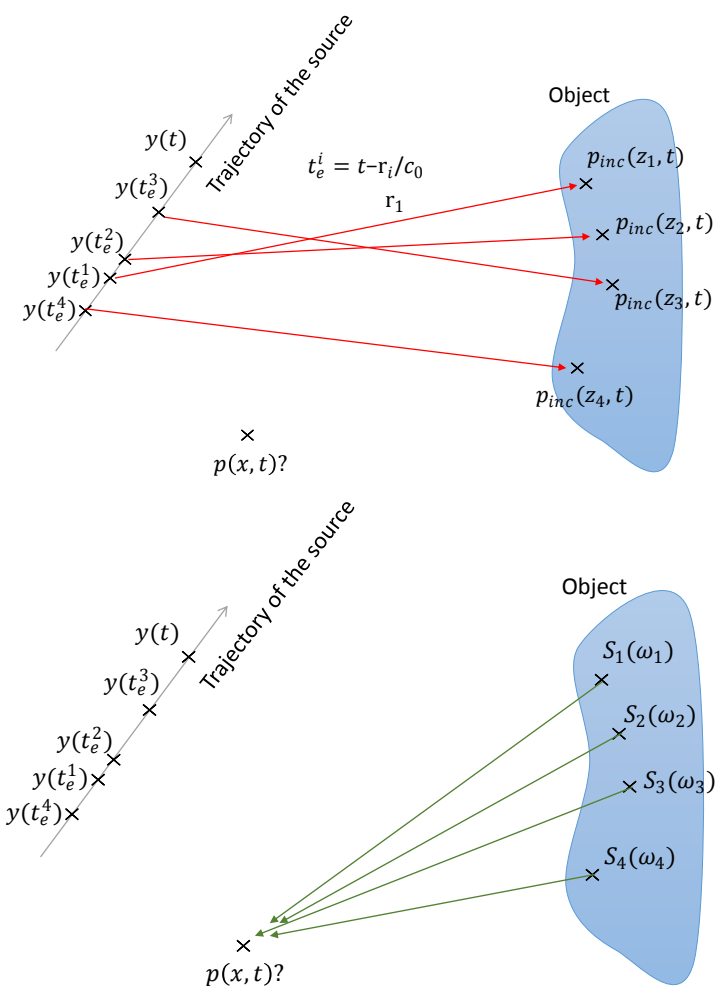

Figure 3: Scattering of the sound wave from a moving source by a rigid object seen as Huygens' equivalent sources, in 2 steps.

The amplitude and frequency of the equivalent sources are dependent on the position and the speed of the source and are computed in the time-domain. The frequency of such a source is given by the Doppler shift applied to the frequency of the source

$$
\omega_{e}=\frac{\omega_{0}}{1-M_{r}} .
$$

The scattered field is then evaluated in a second step, by considering the propagation of sound from the object with its equivalent sources to the listener in the frequency domain. This is only valid under these two hypothesis: 
- The source is moving slowly enough so that a listener on the object hears locally at a given time a harmonic wave.

- The distance from the source to the object is much larger than the distance from the object to the listener so that the propagation time from the object to the listener can be neglected, or the speed of the source is small enough to consider it fixed during the propagation from the object to the listener.

A possible improvement to extend this study beyond the scope of the second hypothesis would be to evaluate the surface pressures at a retarded time which can for example correspond to the propagation time between the source and the geometric center of the object.

Mathematically, Figure 3 translates as

$$
\begin{array}{r}
p(\mathbf{x}, t)=p_{\text {inc }}(\mathbf{x}, t)+\oiint_{\mathcal{S}} 2 p_{\text {inc }}(\mathbf{z}, t) \nabla_{z} \hat{G}\left(\mathbf{z}, \mathbf{x}, \mathbf{k}_{z}\right) \cdot \mathbf{n}_{\text {out }} \\
\cdot \mathcal{D}\left(\mathbf{z}, \mathbf{y}\left(t_{e}\right)\right) d S_{z}
\end{array}
$$

where $p_{\text {inc }}$ is given by Eq. (18) and where $t_{e}$ is the retarded time introduced earlier. The wave number $\mathbf{k}_{\mathbf{z}}$ is the perceived wave number.

\subsection{Multiple reflections of the sound wave emitted by a moving point source}

If the source is moving, the problem of multiple scattering becomes more complex than in the case of a fixed source. A possible way to deal with this problem is to adopt an intuitive approach based on equivalent sources as in Section 3.3 . This method has been implemented in the code and is explained here on the example of two objects and two orders of scattering, as shown in Figure 4. The principle is the following. The source

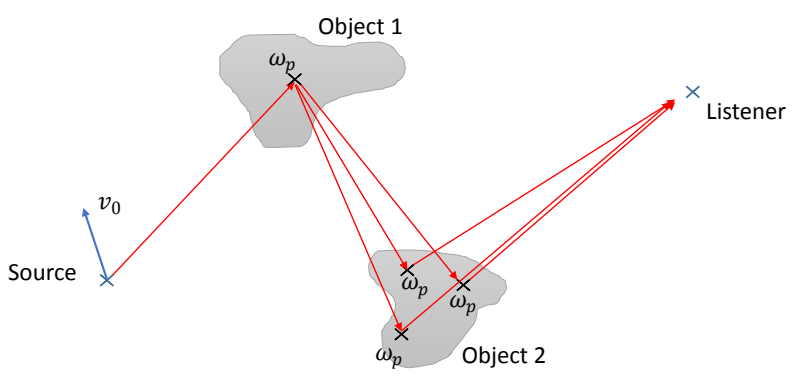

Figure 4: Interpretation of multiple scattering of a single ray from a moving source up to a listener.

emits a sound wave that reaches Object 1 . If the speed of the source is not too large, each point on the surface of this object will see a harmonic wave of frequency $\omega_{p}$ given by Eq. (19). Using the Huygens principle, each of these point can be considered as a fictive source emitting a pressure wave at its own perceived frequency. This wave will reach the second object, where this time all the points will hear a sound at the same frequency, since the objects are fixed relatively to each other. Then these fictitious equivalent sources on Object 2 will emit a sound of still the same frequency $\omega_{p}$ reaching the receiver. In a more general form, the expression for the total pressure at the listener could be derived from Eqs. (10), (16) and (17) by introducing the shifted frequencies and the retarded time and by replacing the expression for the incident pressure by Eq. (18). As each integration point on the objects are considered as individual sources of their own perceived frequency, the computational cost increases exponentially with the level of scattering. Therefore only two levels of scattering are currently implemented in the method for the case of a moving source.

\section{Validation}

In this section we discuss three cases of validation of our implementation of the Kirchhoff-Helmholtz equation targeted to an application to sound reflection on building façades from a moving point source. The first case is a study of the scattering of a plane wave by a rigid sphere for which the analytical solution is well-known. The second case analyses the scattering of a spherical harmonic sound wave by a flat rigid panel for which experimental results were obtained by Cox and Lam [17]. The last case focuses on the sound field generated by a moving harmonic monopole source passing in front of two infinitely high buildings where results are compared with results obtained by a higher-order finite difference time-domain solver (FDTD) [23, 24, 25].

\subsection{Scattering of a plane wave by a sphere}

In this section we focus on a test benchmark for the use of the Kirchhoff approximation to compute scattering by a single object. The idea here is to validate this approximation and its implementation in a well-known case, where an analytical solution can be derived. Scattering of a plane wave by a sphere is a well documented problem [26] that allows comparison with an analytical solution.

Configuration. To simulate an incoming plane wave, a point source is placed far enough from the sphere so that the variations of amplitude of the incident pressure in the area of interest can be neglected. The sphere center is located at $(0,0,0)$ and the spherical source at $\left(-10^{9}, 0,0\right) \mathrm{m}$. In this configuration, the variation of amplitude of the incident pressure within a sphere of $100 \mathrm{~m}$ around the origin is less than $1.10^{-5} \%$. Pressure is computed on a circle of given radius $R$ around the sphere as described in Figure 5. The surface mesh for the sphere is generated with the Matlab package DistMesh developed by Persson [27].

Analytical solution. Based on the works of Morse [28] and Faran [26] the scattered pressure $p_{s c}$ can be written as a series of spherical functions

$$
p_{s c}(r, \theta)=\sum_{n=1}^{\infty} c_{n}\left(j_{n}(k r)-\mathrm{j} n_{n}(k r)\right) P_{n}(\cos \theta)
$$

where $j_{n}$ is the spherical Bessel function of the first kind of order $n, n_{n}$ is the spherical Bessel function of the second kind of order $n, P_{n}$ is the Legendre polynomial of order $n$ and $\mathrm{j}$ is 


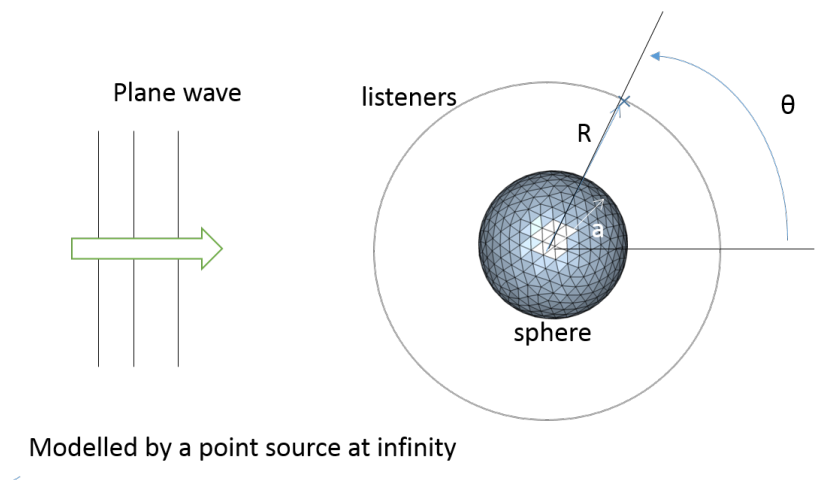

Figure 5: Positions of the source, sphere and listeners for the benchmark of the simulation of scattering of a plane wave by a sphere.

the unit imaginary number. The coefficient $j_{n}-j n_{n}$ ensures that all the waves are outgoing. After applying the hard wall boundary condition on the surface of the sphere and after simple manipulations on the spherical functions, the coefficients $c_{n}$ are found as

$$
c_{n}=-p_{0}(2 n+1)(-j)^{n} \frac{j_{n}^{\prime}(k a)}{2 j_{n}^{\prime}(k a)-h_{n}^{\prime}(k a)}
$$

where the derivatives $j_{n}^{\prime}$ and $h_{n}^{\prime}$ of the spherical Bessel and Hankel function of the first kind can be expressed as

$$
\left[\begin{array}{l}
j_{n}^{\prime}=\frac{1}{2 n+1}\left(n j_{n-1}-(n+1) j_{n+1}\right) \\
h_{n}^{\prime}=\frac{1}{2 n+1}\left(n h_{n-1}-(n+1) h_{n+1}\right) .
\end{array}\right.
$$

Results. Results obtained with the Kirchhoff approximation and analytical results are compared for various values of the Helmholtz number $k a$ and for various listener radii. Results for $R=3 a$ and $k a=3,10$ and 30 are presented in Figure 6 . As expected, the accuracy of the predicted field increases with frequency. For high $k a$, the scattered field is very close to the analytical solution. For small $k a$ the accuracy decreases but the interference pattern is still well predicted. These directivity plots seem to show that the scattered field is better predicted in the backward direction $\left(90^{\circ}<\theta<270^{\circ}\right)$ than in the forward direction $\left(-90^{\circ}<\theta<90^{\circ}\right)$. This appears more clearly when looking at the average error between the predicted values of total pressure and the analytical solution for varying $k a$ and $R$ (Figure 7), in the forward and backward directions. For a given radius, the average error is always higher in the forward direction. In the backward direction, for $k a>5$, the average error is less than $1 \mathrm{~dB}$ and drops to less than $0.5 \mathrm{~dB}$ for $k a>15$.

These results show firstly that the Kirchhoff approximation is well implemented, secondly that the Kirchhoff approximation can be used for high-frequency scattering for values of $\mathrm{ka}$ superior to 3 at least which confirms the results that can be found in the literature. They also show that the use of the Kirchhoff approximation is better suited to compute backward scattering than forward scattering. However, the example of the sphere alone is not sufficient to show that it can be used for sound scattering by buildings, for a sphere has no edges. Another test has to be led and this is the purpose of the next section.

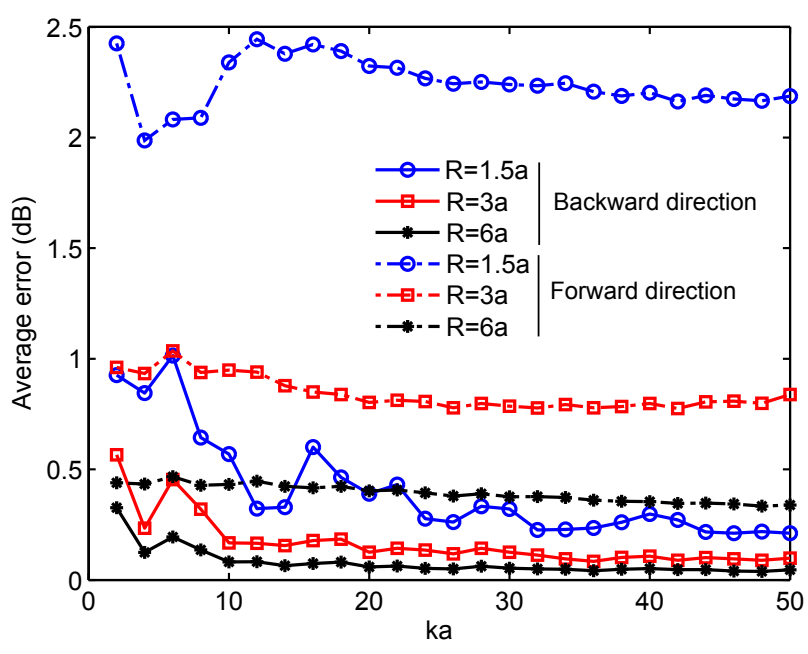

Figure 7: Average error in $\mathrm{dB}$ between the predicted values of total pressure and the analytical solution for varying $k a$ for $R=1.5 a, 3 a$ and $6 a$. In solid line, backward direction $\left(90^{\circ}<\theta<270^{\circ}\right)$ and in dashed line, forward direction $\left(-90^{\circ}<\theta<90^{\circ}\right)$. The average error is computed as the mean value of $\mid$ $20 \log \left(P / P_{\text {anal }}\right) \mid$ for each direction.

\subsection{Scattering of a spherical wave by a rigid panel}

The purpose of this section is to validate the use of the Kirchhoff approximation for sound scattering by an object with flat surfaces and sharp edges, similar to a building façade. Cox and Lam [17] studied the scattering of a spherical sound wave by rigid reflectors and got experimental results for various frequencies. Their study focused mainly on room acoustics, but after scaling of the length and frequencies it is noticeable that it can also apply to vehicle noise and scattering by buildings as the Helmholtz numbers are of the same order. These test cases are therefore interesting in order to quantify the validity of the Kirchhoff approximation for the reflection of sound on a building façade. The set up for the measurements is presented in Figure 8. The measured panel has dimensions $L, H$ and $e$

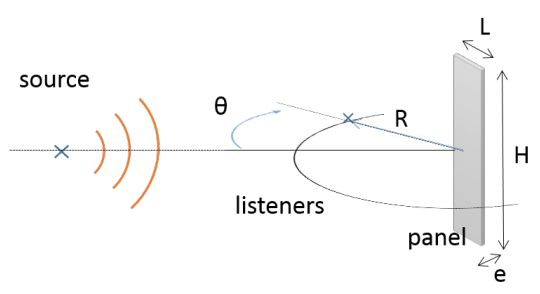

Figure 8: Positions of the source, panel and observers for the benchmark of the simulation of scattering of a plane wave by a rigid panel.

of respectively $0.302,1.92$ and $0.010 \mathrm{~m}$. The source is located at $3.96 \mathrm{~m}$ from the panel, and the pressure is measured along an array of listeners located on a semicircle on the source side at $1.178 \mathrm{~m}$ from the center of the panel. The experimental results are compared with predictions from our Kirchhoff method in Figure 9 and Figure 10 for 2012 and $3995 \mathrm{~Hz}$ respectively. We can see that for small angles of scattering the Kirchhoff approximation gives an accurate prediction of the scattered field. In both cases the scattering pattern is well predicted. For 2012 


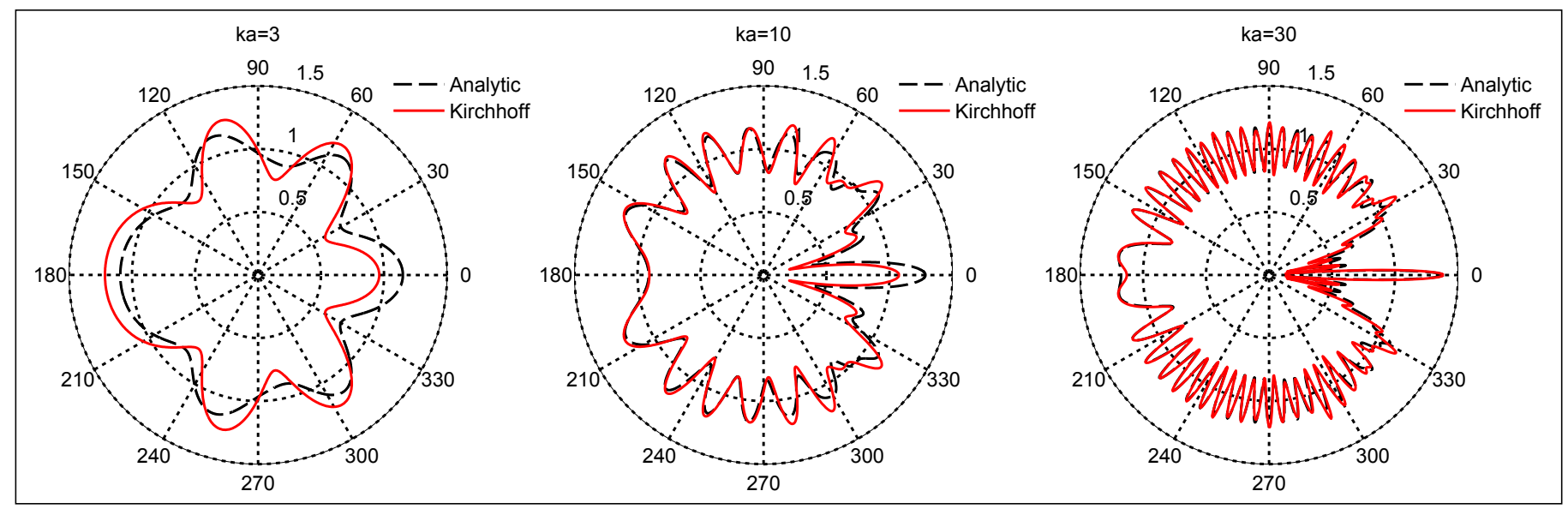

Figure 6: Total acoustic pressure amplitude normalized by incident value at $R=3 a$ for $k a=3,10$ and 30 .

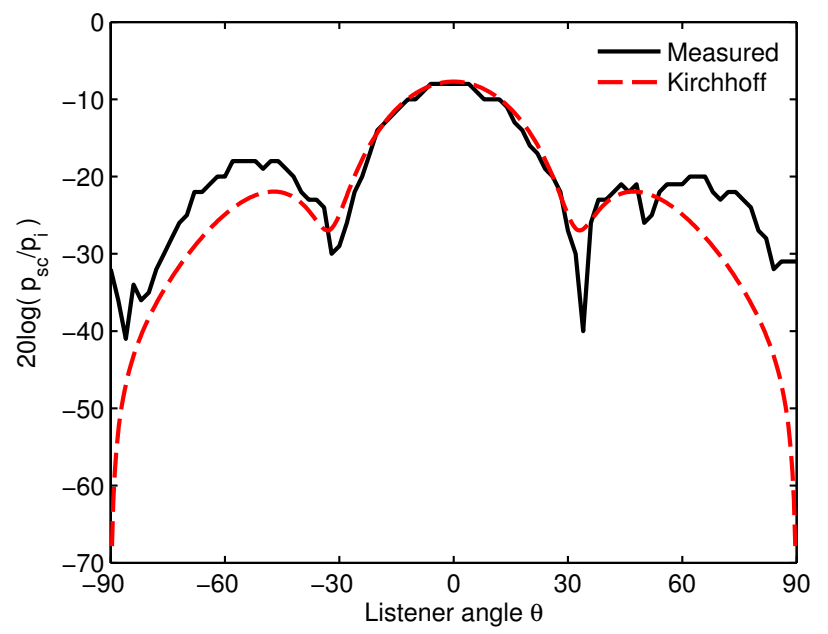

Figure 9: Scattered field, comparison between the results obtained with the Kirchhoff approximation and results measured by Cox and Lam [17] for 2012 $\mathrm{Hz}$.

$\mathrm{Hz}$, good results are obtained for angles between $\pm 30-50^{\mathrm{deg}}$, and for $3995 \mathrm{~Hz}$ good results are obtained for angles between $\pm 60^{\mathrm{deg}}$. This confirms that the Kirchhoff approximation gives better results for high frequencies and small angles of scattering, and more importantly that it can predict accurately the scattered field under these circumstances.

\subsection{Moving point source passing by two buildings}

In this section we present the results of a study of the propagation of a sound wave emitted by a moving point source passing by two rigid buildings. The configuration is presented in Figure 11. The two buildings have horizontal sections of $L=20$ $\mathrm{m}$ by $l=10 \mathrm{~m}$ and they are separated by $s=8 \mathrm{~m}$. They are assumed to have flat rigid surfaces. The source is a monopole point source of frequency $87.5 \mathrm{~Hz}$ travelling at constant speed $v_{0}=60 \mathrm{~m} \cdot \mathrm{s}^{-1}$ in the $z=0$ plane, at a distance $d=10 \mathrm{~m}$ from the front façades of the buildings. At this speed, the Doppler effect causes a shift in the perceived frequency of up to around $20 \%$ for observers facing the source. In these conditions the

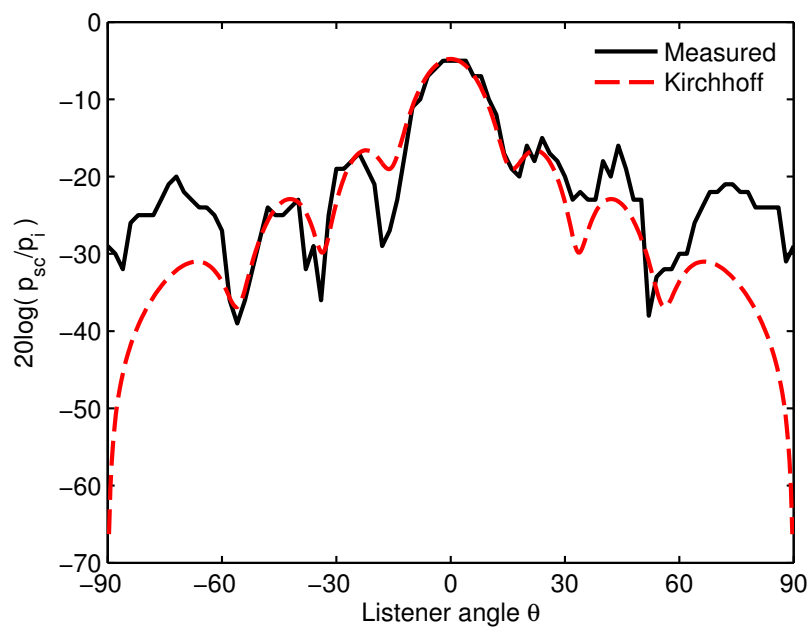

Figure 10: Scattered field, comparison between the results obtained with the Kirchhoff approximation and results measured by Cox and Lam [17] for 3995 $\mathrm{Hz}$.

movement of the source has to be treated in a non quasi-static fashion which justifies the use of this method. The surrounding flow field consists of homogeneous isotropic air at rest, with a sound speed of $346 \mathrm{~m} . \mathrm{s}^{-1}$. The ground is not modelled. Instead, the buildings extend in both $+z$ and $-z$ direction. The results obtained with our method are compared with results obtained with a FDTD method, that are considered as reference results. In the $z$ direction, the buildings are extended up to the outer boundary of the domain for the FDTD method. They have a finite vertical height of $60 \mathrm{~m}$ for our Kirchhoff method - high enough so that the edge effects from the top and bottom edges of the buildings have a negligible contribution in the $z=0$ plane, in which the results are analyzed. In these conditions the buildings can be considered as infinitely tall in both simulations. The domain for the FDTD code extends from -10 to $80 \mathrm{~m}$ in the $x$ direction, from -10 to $70 \mathrm{~m}$ in the $y$ direction and from -50 to $50 \mathrm{~m}$ in the $z$ direction. A sponge zone is set at each outer boundary and is adjusted to ensure very low reflections. A uniform structured mesh was used with 10 points per wavelength. The source has 


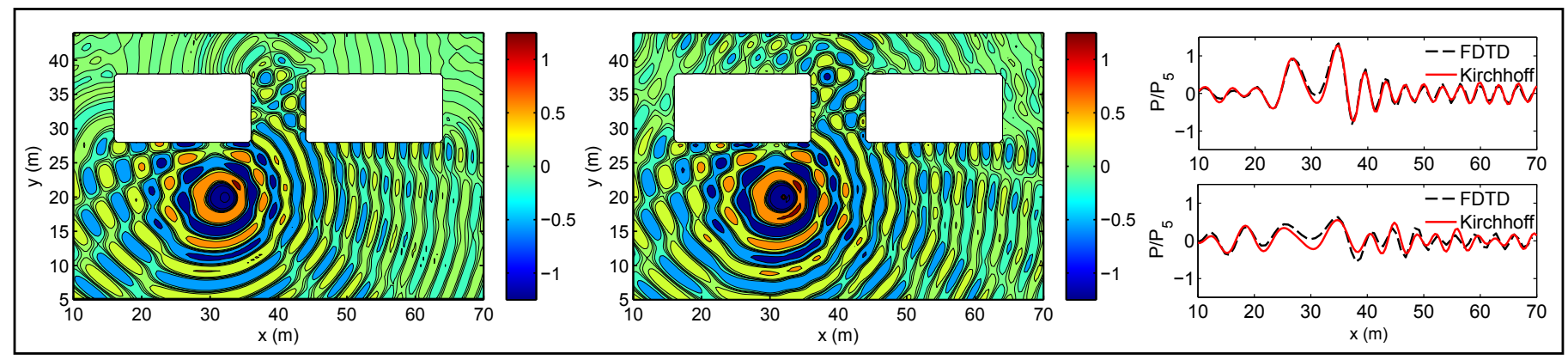

Figure 12: Acoustic pressure field at time $0.200 \mathrm{~s}$ for the FDTD solver (left) and our method (middle) for a moving source of frequency $87.5 \mathrm{~Hz}$, acoustic pressure along the line $y=26 m$ (top right), acoustic pressure along the line $y=10 \mathrm{~m}$ (bottom right). Values are normalized by the pressure amplitude at $5 \mathrm{~m}$ from the source.

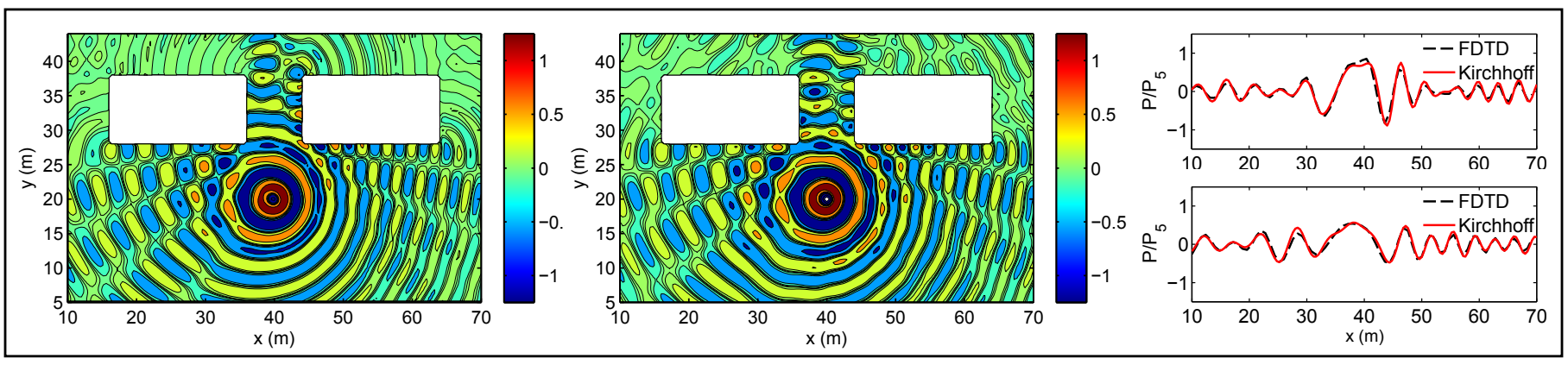

Figure 13: Acoustic pressure field at time $0.334 s$ for the FDTD solver (left) and our method (middle) for a moving source of frequency $87.5 \mathrm{~Hz}$, acoustic pressure along the line $y=26 m$ (top right), acoustic pressure along the line $y=10 m$ (bottom right). Values are normalized by the pressure amplitude at $5 \mathrm{~m}$ from the source.

been matched in amplitude and phase in an empty domain so that a fixed source at $(20,20,0) \mathrm{m}$ gives the same amplitude at the listener position $(40,30,0) \mathrm{m}$ for both methods. For both simulations the source is located at $(20,20,0) \mathrm{m}$ at time $t=0$ and moves along the $y=20 \mathrm{~m}$ line towards increasing $\mathrm{x}$.

The fields of acoustic pressure at times $0.200 \mathrm{~s}$ and $0.334 \mathrm{~s}$,

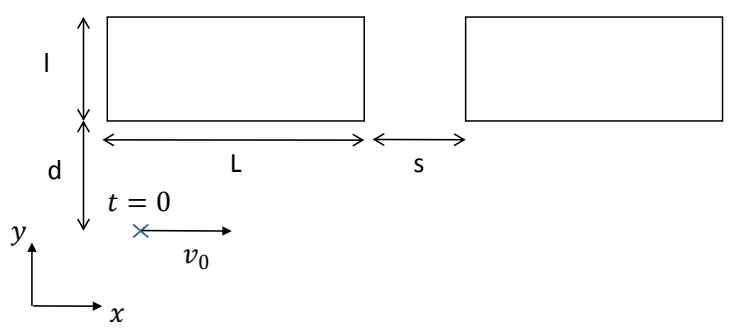

Figure 11: Positions of the source and buildings in the $z=0$ plane for validation against FDTD method.

respectively, are represented in Figures 12 and 13 together with the pressure along the lines $y=10 \mathrm{~m}$ and $y=26 \mathrm{~m}$.

On the whole the agreement between the two methods is good. Both methods give similar patterns for interferences and local maxima and minima of the pressure field are well represented. Some differences can be observed locally, in particular between the two buildings where multiple scattering from the edges strongly affects the field. Indeed multiple reflections are only accounted up to order 2 in our solver whereas a higher number of reflections occurs in the FDTD solver. The extracted pressures on the lines $y=10 \mathrm{~m}$ and $y=26 \mathrm{~m}$ show a good agreement between both methods, the agreement being better closer to the building façades.

The simulations presented in Figures 12 and 13 have been computed with two orders of scattering in the Kirchhoff-based method. As the main focus here is the sound field in the street where the source is moving, the first order might be sufficient and can drastically reduce the computation time. The acoustic pressure field as predicted at time $0.200 s$ with the Kirchhoff method with only one level of reflection on the building façades is represented in Figure 14. The values along the lines $y=10 \mathrm{~m}$ and $y=26 \mathrm{~m}$ are very close to the ones with two orders of reflection represented in Figure 12 (right) and the difference would not be visible on such plots. They are therefore not shown here. As expected, the only improvement brought by a second level of reflection concerns the acoustic field between the two buildings. The Kirchhoff method with only one level of scattering is very interesting here because it produces similar results with a much faster computational time. To visualize the reflections of second order, the substraction of the acoustic field with one and two levels of reflection is represented in Figure 15 The reflected wave on the right façade of the left building after reflection on the left façade of the right building is clearly noticeable.

For these computations, a triangular mesh with 2 points per wavelength was used on the surface of the buildings, and the numerical integration was performed using a 3 points GaussLegendre quadrature, yielding a total of 4 points per wave- 


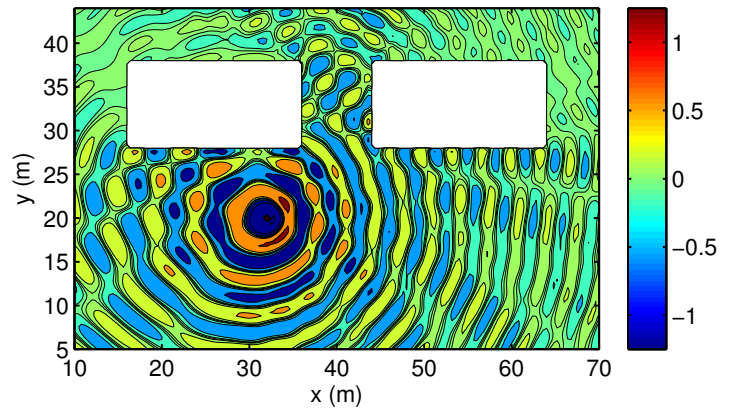

Figure 14: Acoustic pressure field at time $0.200 s$ as predicted by the Kirchhoff method with one order of reflection on the building façades.

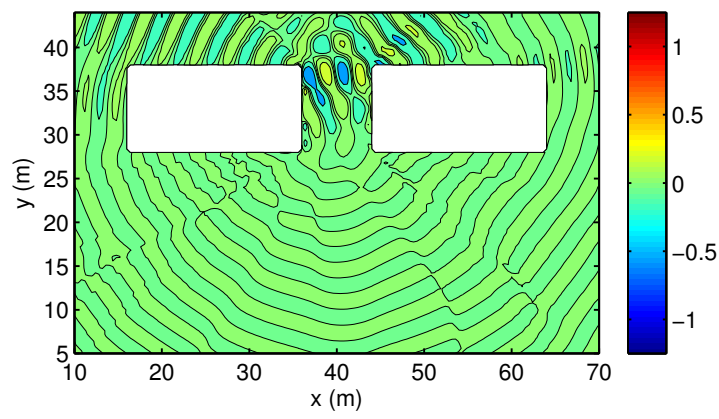

Figure 15: Difference between second order and first order reflection in the acoustic pressure field at time $0.200 s$ as predicted by the Kirchhoff method.

length. It has been observed that results were improved with a finer mesh, at the expense of computational cost. The graphs presented here show that good results can be obtained with a relatively coarse mesh.

It can be of interest to look at the values of surface pressure which, for a first order scattering, are only due to the incident field. The acoustic surface pressure computed at the first level of scattering (incident pressure from the source only) as predicted at time $0.200 \mathrm{~s}$ with the Kirchhoff method is represented in Figure 16. Only the three façades that are needed in the computation are represented. The surfaces in the shadow areas have a zero pressure value, and the movement of the source is clearly visible on the right façade of the left building. Indeed, at time 0.334 s the source has just passed the second edge of this building (the edge along the line $(36,28, z) \mathrm{m}$ ) and only the closest parts of the right façade to the source have received the sound wave coming from the source.

The FDTD method and the Kirchhoff method are really different by nature and it is interesting to see that the results are so similar. Our method produces results which are more approximate than a BEM or a FDTD method but that has the advantage of being less computationally demanding. The loss in information due to the approximations is small and the results are accurate enough to get a good idea of the sound level as the source passes by the two buildings.
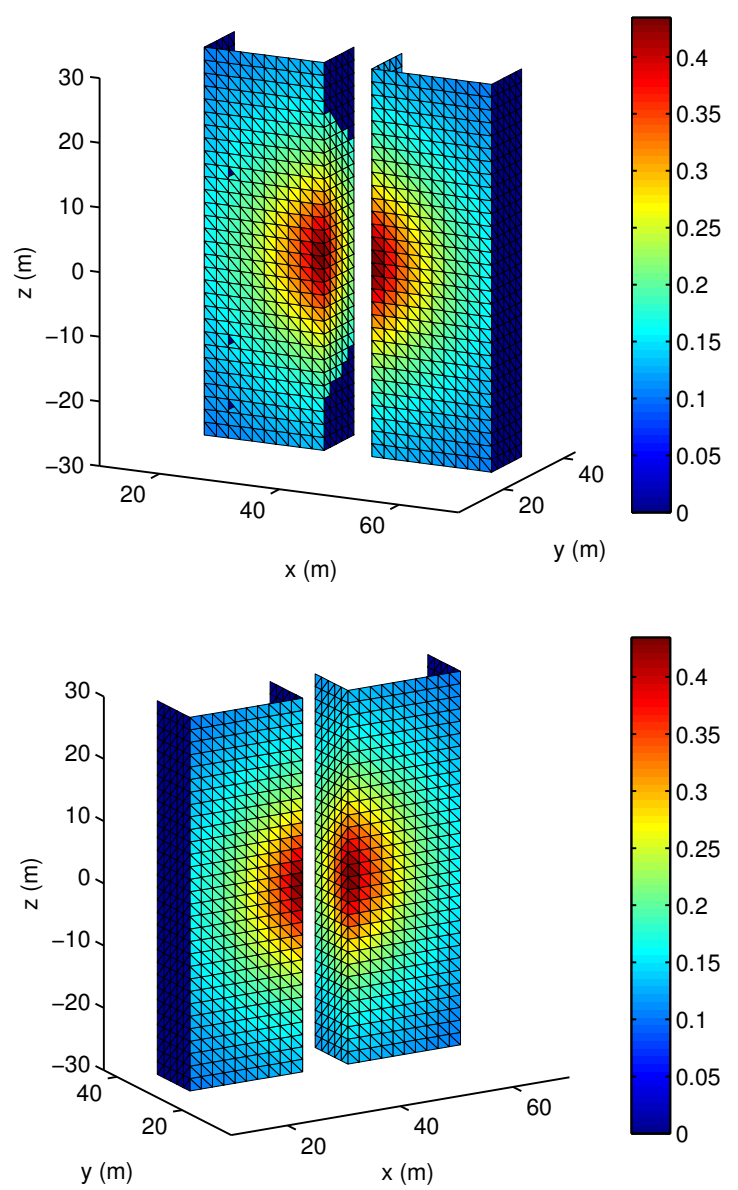

Figure 16: Absolute value of surface acoustic pressure at time $0.334 s$ as predicted by the Kirchhoff method with one order of reflection on the building façades.

\section{Conclusion}

This paper presents an approximate method to compute the sound field generated by a moving harmonic monopole point source taking into account scattering by nearby objects. This method is based on the Kirchhoff-Helmholtz integral equation applying the Kirchhoff approximation. Multiple scattering is accounted for by successive evaluations of the surface pressures and the movement of the source is accounted for both in the expressions of the incident and the scattered field by the introduction of the Doppler shift and the retarded time. This method has been evaluated in three test cases:

- The scattering of a plane wave by a rigid sphere where an analytical solution can be derived.

- The scattering of a spherical sound wave by a rigid plane panel where numerical and experimental results exist.

- The scattering of a moving point source by two adjacent tall buildings where reference results have been obtained with a higher-order FDTD solver.

Results have been shown to be good for high frequency scattering from one object and comparable with results from a FDTD 
method for a moving source passing by two tall buildings, when the focus of study is the sound field on the side of the source. Future work will include further validation of the code and implementation of the reflection from the ground. More realistic sound sources should then be modelled to represent the sound field of a passing vehicle more accurately.

\section{Acknowledgements}

The work presented here is part of the project: "Noise Propagation from Sustainable Vehicle Concepts" led at the Centre for $\mathrm{ECO}^{2}$ Vehicle Design, a VinnExcellence Centre performing multi-vehicular multidisciplinary research to support a sustainable vehicle design development. The authors would like to thank Daniel J. Bodony from the University of Illinois for providing the FDTD code used in the validation process for the moving source case.

\section{References}

[1] European Commission . The EU policy on environmental noise. 2014. URL: http://ec. europa.eu/environment/noise/home.htm [Online; accessed 23-October-2014].

[2] European Commission . Development of a methodology to assess population exposed to high levels of noise and air pollution close to major transport infrastructure. 2006. URL: http://ec.europa.eu/ environment/air/transport/pdf/final_report_main.pdf] [Online; accessed 23-October-2014].

[3] Barry TM, Reagan JA. FHWA highway traffic noise prediction model, fhwa-rd-77-108 final rpt. 1978.

[4] Calculation of road traffic noise, department of transport, welsh office, HMSO. 1988.

[5] Defrance J, Salomons E, Noordhoek I, Heimann D, Plovsing B, Watts $\mathrm{G}$, et al. Outdoor sound propagation reference model developed in the european harmonoise project. Acta Acust un Acust 2007;93(2):213-27.

[6] Kragh J, Plovsing B, Storeheier S, Taraldsen G, Jonasson H. Nordic environmental noise prediction methods, Nord2000 summary report. general nordic sound propagation model and applications in source-related prediction methods. 2002.

[7] Yamamoto K. Road traffic noise prediction model "asj rtn-model 2008": report of the research committee on road traffic noise. Acoust Sci Technol 2010;31(1):2-55. doi $10.1250 /$ ast. 31.2

[8] ISO 9613-2, acoustics - attenuation of sound during propagation outdoors - part 2: General method of calculation. 1996.

[9] Garg N, Maji S. A critical review of principal traffic noise models: Strategies and implications. Environ Impact Assess Rev 2014;46(0):68-81. doi $10.1016 /$ j.eiar.2014.02.001

[10] Attenborough K, Li KM, Horoshenkov K. Predicting outdoor sound. CRC Press; 2006.

[11] Remillieux MC, Corcoran JM, Haac TR, Burdisso RA, Svensson UP. Experimental and numerical study on the propagation of impulsive sound around buildings. Appl Acoust 2012;73(10):1029-44. doi 10.1016/j. apacoust.2012.04.011

[12] Hornikx M, Waxler R, Forssén J. The extended fourier pseudospectral time-domain method for atmospheric sound propagation. J Acoust Soc Am 2010;128(4):1632-46.

[13] Marburg S, Nolte B, Bernhard R, Wang S. Computational acoustics of noise propagation in fluids: finite and boundary element methods. Springer; 2008.

[14] Heutschi K. Calculation of reflections in an urban environment. Acta Acust un Acust 2009;95(4):644-52. doi 10.3813/AAA . 918193

[15] Tinetti AF, Dunn MH. Aeroacoustic noise prediction using the fast scattering code. AIAA Paper 2005;3061.

[16] Baker BB, Copson ET. The mathematical theory of Huygens' principle. Chelsea Publishing Company; 1950.
[17] Cox TV, Lam Y. Evaluation of methods for predicting the scattering from simple rigid panels. Appl Acoust 1993;40(2):123 -40. doi 10.1016/ 0003-682X(93) 90086-L

[18] Foote KG, Francis DTI. Comparing kirchhoff-approximation and boundary-element models for computing gadoid target strengths. J Acoust Soc Am 2002;111(4):1644-54. doi 10.1121/1.1458939

[19] Eaton DW. Backscattering from spherical elastic inclusions and accuracy of the kirchhoff approximation for curved interfaces. Geophys J Int 2006;166(3):1249-58. doi 10.1111/j .1365-246X.2006.03047.x

[20] Jebsen GM, Medwin H. On the failure of the kirchhoff assumption in backscatter. J Acoust Soc Am 1982;72(5):1607-11. doi 10.1121/1. 388496

[21] Wirgin A. Scattering from sinusoidal gratings: an evaluation of the kirchhoff approximation. J Opt Soc Am 1983;73(8):1028-41. doi 10.1364/ JOSA.73.001028

[22] Åbom M. An introduction to flow acoustics. Trita-AVE; 2008.

[23] Kim J, Bodony DJ, Freund J. Adjoint-based control of loud events in a turbulent jet. J Fluid Mech 2014;741:28-59. doi 10.1017/jfm.2013. 654

[24] Bodony DJ. Accuracy of the simultaneous-approximation-term boundary condition for time-dependent problems. J Sci Comput 2010;43(1):11833. doi $10.1007 / \mathrm{s} 10915-010-9347-4$

[25] Bodony D, Zagaris G, Reichert A, Zhang Q. Provably stable overset grid methods for computational aeroacoustics. J Sound Vib 2011;330:416179.

[26] Faran JJ. Sound scattering by solid cylinders and spheres. J Acoust Soc Am 1951;23(4):405-18. doi 10.1121/1.1906780

[27] Persson PO, Strang G. A simple mesh generator in matlab. SIAM Review 2004;46(2):329-45.

[28] Morse PM. Vibration and Sound. McGraw-Hill Book Company Inc.; 1948. 